\title{
HURWITZ PAIRS AND CLIFFORD VALUED INNER PRODUCTS
}

\author{
JAN CNOPS \\ Vakgroep Wiskundige Analyse, RUG \\ Galglaan 2, B-9000 Gent, Belgium \\ E-mail: jc@cage.rug.ac.be
}

\begin{abstract}
After an overview of Hurwitz pairs we are showing how to actually construct them and discussing whether, for a given representation, all Hurwitz pairs of the same type are equivalent. Finally modules over a Clifford algebra are considered with compatible inner products; the results being then aplied to Hurwitz pairs.
\end{abstract}

Introduction. Hurwitz pairs appear under different disguises in mathematical physics and are often introduced in a rather ad hoc way. It has been proved by several authors however that a Hurwitz pair is nothing else than an irreducible representation of a Clifford algebra with an inner product compatible with the main antiautomorphism (see e.g. [10] and [9]), and this links the notion of Hurwitz pair to the study of sesquilinear forms on spinor spaces. In the first section of this paper an overview is given of this relation.

This result however does not show how to actually construct Hurwitz pairs, nor does it tell whether, for a given representation, all Hurwitz pairs of the same type are equivalent. The answer to this question is yes, and both problems are dealt with in the second section.

In the final section modules over a Clifford algebra are considered with a compatible inner product (whithout the demand of irreducibility). It is proved that such inner products can be considered as the trace of Clifford valued inner products, a result which is then applied to Hurwitz pairs.

\section{Definitions and notations}

Clifford algebras. Let $\mathbb{R}^{p q}$ be the real $n$-dimensional space, where $n=p+q$, endowed with the nondegenerate inner product $\mathcal{B}(\cdot, \cdot)$ of signature $(p, q)$. We assume that

1991 Mathematics Subject Classification: Primary 15A66, Secondary 30 G35.

Post-doctoral researcher, N.F.W.O., Belgium.

The paper is in final form and no version of it will be published elsewhere. 
an orthonormal basis $e_{1}, \ldots, e_{n}$ is given, that is a basis such that

$$
\begin{array}{ccc}
\mathcal{B}\left(e_{i}, e_{i}\right)= & -1, & i=1, \ldots, p \\
\mathcal{B}\left(e_{i}, e_{i}\right)= & 1, & i=p+1, \ldots, n \\
\mathcal{B}\left(e_{i}, e_{j}\right)= & 0, & i \neq j
\end{array}
$$

It should be noticed that the notation is taken such that Euclidean space with positive definite inner product is written as $\mathbb{R}^{0, n}$.

The Clifford algebra over $\mathbb{R}^{p q}$ is denoted by $\mathbb{R}_{p q}$. It is the free algebra generated by the $e_{i}$ modulo the relations

$$
e_{i} e_{j}+e_{j} e_{i}=-2 \mathcal{B}\left(e_{i}, e_{j}\right)
$$

Elements of $\mathbb{R}_{p q}$ are called Clifford numbers or Clifford scalars. The Clifford algebra has a basis (as vector space over $\mathbb{R}$ ) of elements $e_{A}$ where $A$ is a subset of $\{1, \ldots, n\}$, and where $e_{\left\{i_{1}, \ldots, i_{k}\right\}}$ is identified with $e_{i_{1}} \ldots e_{i_{k}}$, at least if $i_{1}<\ldots<i_{k}$. If $A$ has $k$ elements, then $e_{A}$ is called a $k$-vector. Likewise any linear combination of $k$-vectors is called a $k$-vector. The elements of $\mathbb{R}^{p q}$ are the 1 -vectors, or vectors, of the Clifford algebra and will be denoted by $\vec{x}, \vec{y}$ etc.

The main antiautomorphism is defined by

$$
\overline{\vec{x}}=-\vec{x} \quad \overline{(a b)}=\bar{b} \bar{a} .
$$

Each Clifford algebra $\mathbb{R}_{p q}$ is isomorphic, either to a full matrix algebra $\mathbb{F}(t)$ or to a double matrix algebra $\mathbb{F}(t) \oplus \mathbb{F}(t)$, where $\mathbb{F}$ is either $\mathbb{C}, \mathbb{R}$ or $\mathbb{H}$, and $t$ is some power of 2. $\mathbb{F}$ will sometimes be called the field of the Clifford algebra $\mathbb{R}_{p q}$. Explicit tables of the field $\mathbb{F}$, the dimension $t$ and whether one needs a full or a double algebra for given $\mathbb{R}_{p q}$ can be found in various places, e.g. in [7].

Matrices and operators. The notation $M^{t}$ is used for the transpose of a matrix, and $M^{-t}$ indicates the inverse of the transpose.

Furthermore generalised inverses will be needed, at least for linear operators on finite dimensional spaces. Let $V$ be such a space, endowed with a euclidean metric, and $K$ be a linear operator. then $K$ is invertible as an operator from $\operatorname{ker}^{\perp} K$ to im $K$. The inverse of this, extended with 0 on im $\mathrm{im}^{\perp} K$ is the generalised inverse, $K^{\dagger}$. Clearly $K^{\dagger} K$ is the orthogonal projection on $\operatorname{ker}^{\perp} K$, and so $K K^{\dagger} K=K$ and $K^{\dagger} K K^{\dagger}=K^{\dagger}$. The identical transformation on a vector space $V$ will be denoted as $I_{V}$; the notation $I_{n}$ will be used for the unit $n \times n$ matrix.

Hurwitz pairs. Consider two vector spaces, $S$ of dimension $n+1$ and $V$ of dimension $t$, both endowed with a bilinear inner product, $(\cdot, \cdot)_{S}$ and $(\cdot, \cdot)_{K}$. (The reason we do not use the notation $(\cdot, \cdot)_{V}$ is that in certain cases it will turn out that different metrics on the same representation will turn out to give different Hurwitz pairs.) Here $(\cdot, \cdot)_{S}$ is symmetric, with signature $(s, r+1)\left((\vec{x}, \vec{y})_{S}=(\vec{y}, \vec{x})_{S}\right)$ and $(\cdot, \cdot)_{K}$ is either symmetric or antisymmetric $\left((v, w)_{K}=\sigma(w, v)_{K}\right.$, where $\left.\sigma= \pm 1\right)$, and both are non-degenerate. Notice again that signature $(s, r+1)$ is to be read as ' $s$ minus signs and $r+1$ plus signs'.

A bilinear mapping $\circ: S \times V \rightarrow V$ is called a Hurwitz multiplication if it satisfies the following conditions (see [4]): 
H.1

$$
(x, x)_{S}(v, w)_{K}=(x \circ v, x \circ w)_{K}
$$

for all $x, v$ and $w$ (this is called the generalised Hurwitz condition).

H.2 There exists $e$ in $S$ such that $e$ o is the identity on $V$.

H.3 There is no subspace of $V$ invariant under $\circ$.

The complete structure is called a Hurwitz pair. If the condition of irreducibility, H.3, is not met, the structure is called a pre-Hurwitz pair. Notice that it automatically follows that $(e, e)_{S}=1$.

1. Classification of Hurwitz pairs. Take $S^{\prime}$ to be the orthogonal complement of e. $S^{\prime}$ has signature $(s, r)$. We shall consider the Clifford algebra $\mathbb{R}_{s r}$ over $S^{\prime}=\mathbb{R}^{s r}$, so the elements of $S^{\prime}$ can be considered as the vectors of the Clifford algebra, and the notation $\vec{x}$, $\vec{y}$ and so on for elements of $S^{\prime}$ can be used; the elements of $S$ are then called paravectors. The following theorem summarises the results of Randriamihamison:

TheOREM 1. Suppose $S, V$ and $\circ$ form a Hurwitz pair. Then

(i) for each $\vec{x}$ in $S^{\prime}$, the adjoint of $\vec{x} \circ$ viewed as an operator on $V$ is $-\vec{x} \circ$.

(ii) The algebra of linear operators generated by the action of elements of $S$ on $V$ is isomorphic to $\mathbb{R}_{\text {sr }}$.

Pr o of. Take $\vec{x}$ in $S^{\prime}$. Applying the definition of a Hurwitz pair to the paravector $e+\vec{x}$ gives

$$
((e+\vec{x}) \circ v,(e+\vec{x}) \circ w)_{K}=(e+\vec{x}, e+\vec{x})_{S}(v, w)_{K}=\left(1+(\vec{x}, \vec{x})_{S}\right)(v, w)_{K}
$$

where the fact that $\vec{x}$ is orthogonal to $e$ is used. On the other hand, by linearity

$$
\begin{gathered}
((e+\vec{x}) \circ v,(e+\vec{x}) \circ w)_{K}=(e \circ v, e \circ w)_{K}+(\vec{x} \circ v, e \circ w)_{K}+ \\
\quad(e \circ v, \vec{x} \circ w)_{K}+(\vec{x} \circ v, \vec{x} \circ w)_{K} \\
=(v, w)_{K}+(\vec{x} \circ v, w)_{K}+ \\
(v, \vec{x} \circ w)_{K}+(\vec{x}, \vec{x})_{S}(v, w)_{K} .
\end{gathered}
$$

Comparison of the two expressions gives $(\vec{x} \circ v, w)_{K}+(v, \vec{x} \circ w)_{K}=0$, which proves (i). It then follows that $(\vec{x} \circ \vec{x} \circ v, w)_{K}=-\mathcal{B}(\vec{x}, \vec{x})(v, w)$ for any $w$ and so that $\vec{x} \circ \vec{x} \circ v=v$ since the inner product is not degenerate. Taking an orthonormal basis $e_{1}, \ldots, e_{s+r}$ of $S^{\prime}$ one then sees that

$$
e_{i} \circ e_{j} \circ+e_{j} \circ e_{i} \circ=-2 \mathcal{B}\left(e_{i}, e_{j}\right)
$$

taking first $\vec{x}=e_{i}$ and then $\vec{x}=e_{i}+e_{j}$. This proves the isomorphism.

Hence we can consider $V$ to be a module over this Clifford algebra, where the action of an element $a$ of $\mathbb{R}_{s r}$ on an element $v$ is denoted as $a v$ in such a way that $\vec{x} v=\vec{x} \circ v$.

A Hurwitz pair can thus be redefined as follows:

Definition. $\left(\mathbb{R}_{s r}, V, m, \kappa\right)$ is called a Hurwitz pair if and only if $m$ is an irreducible representation of $\mathbb{R}_{s r}$ on $V$ and $\kappa$ defines an inner product on $V$ such that

H.1' $(m(a) v, w)_{\kappa}=(v, m(\bar{a}) w)_{\kappa}$.

Corollary 2. All Hurwitz pairs are given by the following table: 


$\begin{array}{lllllllll}r \backslash s & 0 & 1 & 2 & 3 & 4 & 5 & 6 & 7 \\ 0 & \text { S } & - & \text { A } & \text { A } & \text { AS } & - & \text { AS } & \text { S } \\ 1 & \text { AS } & \text { A } & \underline{A} & \text { A } & \text { AS } & \text { AS } & \underline{\text { AS }} & \text { AS } \\ 2 & \text { AS } & \text { A } & \text { A } & - & \text { S } & \text { S } & \text { AS } & - \\ 3 & \underline{\text { AS }} & \text { AS } & \text { AS } & \text { S } & \underline{\text { S }} & \text { S } & \text { AS } & \text { AS } \\ 4 & \text { AS } & - & \text { AS } & \text { S } & \text { S } & - & \text { A } & \text { A } \\ 5 & \text { AS } & \text { AS } & \underline{\text { AS }} & \text { AS } & \text { AS } & \text { A } & \text { A } & \text { A } \\ 6 & \text { S } & \text { S } & \text { AS } & - & \text { AS } & \text { A } & \text { A } & - \\ 7 & \underline{\text { S }} & \text { S } & \text { AS } & \text { AS } & \underline{\text { AS }} & \text { AS } & \text { AS } & \text { S }\end{array}$

P r o of. The table gives all possibilities for sesquilinear forms on irreducible representations as given in [8].

The table has to be read as follows:

First of all $s$ and $r$ are given modulo 8. An $\mathrm{S}$ in the appropriate box means there is a symmetric inner product, an A that there is an antisymmetric one. The cases where there are two inequivalent representations are underlined. As an example take Minkowski space with signature $(-+++)$. Then $S^{\prime}$ has signature $(-++)$, and so $s=1$ and $r=2$. There exists a Hurwitz pair, and it has an antisymmetric inner product.

It should be noticed that in the cases where no Hurwitz pair exists it is still possible to find a $V$ which is the direct sum of two spinor spaces such that H.1-H.2 hold, but irreducibility is of course lost.

If $V$ is given coordinates, then of course $m$ can be thought of as a matrix representation, while $\kappa$ can be thought of as a matrix defining the inner product in the classical way.

\section{Construction of Hurwitz pairs}

Matrix representations. In order to construct the Hurwitz pairs matrix representations will be needed. In the sequel we assume that $V$ is a irreducible module over $\mathbb{R}_{s r}$. If we introduce coordinates on $V$ we have the matrix representation $m$ associating with $a \in \mathbb{R}_{s r}$ the matrix $m(a)$. It should be emphasised that we always regard $V$ as a vector space over $\mathbb{R}$ and so the matrices considered will be real matrices. The metric $K$ can be represented by a matrix $\kappa$, with $\kappa^{t}=\sigma \kappa$ where $\sigma= \pm 1$, and the notation $(\cdot, \cdot)_{\kappa}$ will be used for the inner product. Since we shall often be needing either different representations of $\mathbb{R}_{s r}$ on $V$ or different inner products the notation $\left(\mathbb{R}_{s r}, V, m, \kappa\right)$ wil be used.

Definition. Let $\left(\mathbb{R}_{s r}, V, m, \kappa\right)$ and $\left(\mathbb{R}_{s r}, W, u, \kappa^{\prime}\right)$ be two Hurwitz pairs over the same Clifford algebra. Then they are called equivalent if and only if there exists an invertible linear mapping $c$ with matrix representation $C$ such that

1. $m(a)=C^{-1} u(a) C$ for all $a \in \mathbb{R}_{s r}$.

2. $(u, v)_{\kappa}= \pm(c(u), c(v))_{\kappa^{\prime}}$ for all $u$ and $v$ in $V$.

Of course one can always identify $V$ with $W$ in a canonical way since both have coordinates. 
A new matrix representation $t$ over $V$ can be found putting

$$
t(a)=m^{t}(\bar{a})
$$

where $m^{t}(a)$ is the transpose of $m(a)$. As $t$ is the composition of two antiautomorphisms and one morphism, it is itself indeed a morphism. Clearly for the inner product defined by $\kappa$ the adjoint for a matrix $M$ is given by $\kappa^{-1} M^{t} \kappa$, and so criterion H.1' (which states that $\bar{a}$ is the adjoint of $a$ as an operator on $V)$ can be translated into $m(\bar{a})=\kappa^{-1} m^{t}(a) \kappa$. It is necessary and sufficient that this be true for all vectors $\vec{x}$ since both $m(a) \rightarrow m(\bar{a})$ and $m(a) \rightarrow \kappa^{-1} m^{t}(a) \kappa$ are antiautomorphisms of the algebra $m\left(\mathbb{R}_{s r}\right)$, and this algebra is generated by the $m(\vec{x})$. The criterion then becomes

$$
-m(\vec{x})=\kappa^{-1} m^{t}(\vec{x}) \kappa .
$$

Before we continue with matrix representations we prove that it doesn't matter which equivalent representation we take to obtain our results. Moreover it is useful to write down the transition formulae for the matrices involved.

Lemma 3. Let $\left(\mathbb{R}_{s r}, V, m, \kappa\right)$ be a Hurwitz pair, and let $\left(\mathbb{R}_{s r}, V, u\right)$ be an equivalent irreducible representation. Then there exists a matrix $\kappa^{\prime}$ such that $\left(\mathbb{R}_{s r}, V, u, \kappa^{\prime}\right)$ is an equivalent Hurwitz pair.

Proof. Since the representations are equivalent there exists a matrix $C$ such that $m(a)=C^{-1} u(a) C$ for all $a$ in the Clifford algebra.

For the vectors $\vec{x} \in \mathbb{R}_{s r}$ we have that

$$
m(\vec{x})=-\kappa^{-1} m(\vec{x})^{t} \kappa .
$$

If we define

$$
\kappa^{\prime}=C^{-t} \kappa C^{-1}
$$

then obviously

$$
\begin{aligned}
u(\vec{x}) & =C m(\vec{x}) C^{-1}=-C \kappa^{-1} m(\vec{x})^{t} \kappa C^{-1} \\
& =-\left(C \kappa^{-1} C^{t}\right)\left(C^{-t} m(\vec{x})^{t} C^{t}\right)\left(C^{-t} \kappa C^{-1}\right)=-\kappa^{\prime-1} u(\vec{x})^{t} \kappa^{\prime} .
\end{aligned}
$$

At this point it is convenient to introduce an orthonormal basis for the vector space $S^{\prime}, e_{1}, \ldots, e_{p}$ such that $\left(e_{i}, e_{i}\right)_{S}=-1$ for $i \leq s,\left(e_{i}, e_{i}\right)_{S}=1$ for $s<i \leq s+r=p$. This basis coincides with the basis for the Clifford algebra $\mathbb{R}_{s r}$, but notice that $e_{i}^{2}=-\left(e_{i}, e_{i}\right)_{S}$. We have proved (corollary 1.3.1) that there exists an irreducible matrix representation $m$ of the Clifford algebra such that

$$
m^{t}\left(e_{i}\right)=e_{i}^{2} m\left(e_{i}\right)
$$

Moreover, for any irreducible representation of a Clifford algebra, one can always find coordinates on $V$ such that this condition is satisfied. We shall call a matrix representation with this property a standard representation. It is easy to formulate an equivalent definition for a Hurwitz pair, now in terms of matrices:

THEOREM 4. Let $\mathbb{R}^{n}$ be endowed with an inner product given by a matrix $\kappa$, where $\kappa^{t}= \pm \kappa$, and let $m: \mathbb{R}_{s r} \rightarrow \mathbb{R}^{n \times n}$ be a standard matrix representation of $\mathbb{R}_{s r}$. Then $\left(\mathbb{R}_{s r}, \mathbb{R}^{n}, m, \kappa\right)$ is a Hurwitz pair if and only if 
M.1 $-e_{i}^{2} \kappa^{-1} m\left(e_{i}\right) \kappa=m\left(e_{i}\right)$.

M.3 $m$ is irreducible.

Pro of. that M.3 is equivalent to H.3' is obvious, and M.1 follows directly from H.1'. We prove that H.1' follows from M.1.

M.1 can be rewritten as $-\kappa^{-1} m^{t}\left(e_{i}\right) \kappa=m\left(e_{i}\right)$, because we have a standard representation, and then as $\kappa^{-1} m^{t}\left(e_{i}\right) \kappa=m\left(\overline{e_{i}}\right)$, since $\overline{e_{i}}=-e_{i}$, which shows that criterion (1) holds.

ThEOREM 5. Let $\left(\mathbb{R}_{s r}, V, m\right)$ be an arbitrary irreducible representation of $\mathbb{R}_{s r}$, with associated matrix representations $m$ and $t$ as defined before. Then there exists a Hurwitz pair $\left(\mathbb{R}_{s r}, V, m, \kappa\right)$ if and only if $m$ and $t$ are equivalent.

To prove this we need a lemma.

LEMMA 6. Let $\kappa$ be a matrix such that $\kappa^{t}=\sigma \kappa$ with $\sigma= \pm 1$ and $\kappa m(\vec{x})=-m(\vec{x})^{t} \kappa$ for all $\vec{x} \in S^{\prime}$. Then $\kappa$ is either zero or invertible.

Proof. The basic idea is to prove that the kernel of $\kappa$ is invariant under $m$, which is a variant of Schur's lemma. Notice that Schur's lemma itself cannot be generalised, since inner products of hyperbolic type exist, and for that case $\kappa$ has two different eigenvalues. Since $m$ is irreducible then either $\operatorname{ker} \kappa=\{0\}$, and $\kappa$ is invertible, or $\operatorname{ker} \kappa=V$, and $\kappa=0$.

Multiplying the equation $\kappa m(\vec{x})=-m^{t}(\vec{x}) \kappa$ with $K^{\dagger}$ gives

$$
K^{\dagger} \kappa m(\vec{x})=-K^{\dagger} m^{t}(\vec{x}) \kappa=-K^{\dagger} m^{t}(\vec{x}) \kappa K^{\dagger} \kappa .
$$

Taking the transpose gives

$$
m^{t}(\vec{x}) \kappa K^{\dagger}=-\kappa K^{\dagger} \kappa m(\vec{x}) K^{\dagger} .
$$

Replacing $m^{t}(\vec{x}) \kappa K^{\dagger}$ in the first equation by this gives $K^{\dagger} \kappa m(\vec{x})=K^{\dagger} \kappa m(\vec{x}) K^{\dagger} \kappa$. Applying the right hand side to a vector $v$ in ker $\kappa$ gives zero, so $m(\vec{x}) v \in$ ker $\kappa$ for all $\vec{x}$. This means that ker $\kappa$ is invariant under $m$.

We can now prove the theorem.

Proof. We know that the representations are equivalent if and only if there exists invertible $C$ with $m(\vec{x})=-C^{-1} m^{t}(\vec{x}) C$. If a Hurwitz pair exists, then $C=\kappa$ satisfies this condition (this is simply eq. (1)). If on the other hand such $C$ exists, it follows that $C m(\vec{x})=-m^{t}(\vec{x}) C$ for any $\vec{x}$, and transposing this, $C^{t} m(\vec{x})=-m^{t}(\vec{x}) C^{t}$. We split $C$ into its symmetric and its antisymmetric part, and prove that at least one of them gives a suitable metric. Putting $C^{ \pm}=C \pm C^{t}$, adding or subtracting the two equations gives $C^{ \pm} m(\vec{x})=-m^{t}(\vec{x}) C^{ \pm}$. According to the lemma both $C^{ \pm}$are either invertible or zero, and their sum, $C$, is not zero, so at least one of them is invertible. That one satisfies all conditions for a $\kappa$ matrix.

The following lemma will be important, not only in the construction of different Hurwitz pairs for a given Clifford algebra, but also in the proof of the equivalence of Hurwitz pairs.

Lemma 7. Let $\left(\mathbb{R}_{s r}, V, m, \kappa_{1}\right)$ and $\left(\mathbb{R}_{s r}, V, m, \kappa_{2}\right)$ be two Hurwitz pairs. Then $\kappa_{j}^{-1} \kappa_{i}$ commutes with the representation $m\left(\mathbb{R}_{\text {sr }}\right)$, where $i, j \in\{1,2\}$. 
Proof. taking equation (1) for $\kappa_{i}$ gives $m(\vec{x})=-\kappa_{i}^{-1} m^{t}(\vec{x}) \kappa_{i}$ and the transpose of (1) for $\kappa_{j}$ gives $m^{t}(\vec{x})=-\kappa_{j}^{t} m(\vec{x}) \kappa_{j}^{-t}$. Inserting the last expression for $m^{t}(\vec{x})$ into the first equation gives $m(\vec{x})=\kappa_{i}^{-1} \kappa_{j}^{t} m(\vec{x}) \kappa_{j}^{-t} \kappa_{i}$. Hence $\kappa_{j}^{-t} \kappa_{i}$ commutes with all the $m(\vec{x})$, and so it commutes with the complete $m\left(\mathbb{R}_{s r}\right)$. But $\kappa_{j}^{-t}$ is (possibly up to sign) $\kappa_{j}^{-1}$.

Remark. An alternative proof can be based on a standard representation $m$ : for such representation $\kappa_{j}$ commutes (anticommutes) with $m\left(e_{k}\right)$ whenever $\kappa_{i}$ does, and so does $\kappa_{j}^{-1}$. Hence $\kappa_{j}^{-1} \kappa_{i}$ commutes with all $m\left(e_{k}\right)$, and so with $m\left(\mathbb{R}_{s r}\right)$.

It is of course quite well possible that the two matrices $\kappa_{i}$, even if they are different, define equivalent Hurwitz pairs. As a matter of fact it will be proved later that this is the case whenever $\kappa_{1}$ and $\kappa_{2}$ are of the same type, i.e. if both are symmetric, or both antisymmetric.

It is now already possible to construct Hurwitz pairs in certain specific cases for which we return to condition M.1. Put in other words it says that $\kappa$ anticommutes with all $m\left(e_{i}\right)$ such that $e_{i}^{2}=1$ and commutes with all $m\left(e_{i}\right)$ such that $e_{i}^{2}=-1$. Hence $\kappa m(\vec{x})=m(T \vec{x}) \kappa$ for all $\vec{x}$, where $T$ is an orthogonal transformation on $\mathbb{R}^{s r}$, and it is logical to look for $\kappa$ of the form $m(a)$, where $a$ is a product of basic vectors $e_{i}$. Indeed, for a product $e_{i_{1}} \ldots e_{i_{t}}$, it is easily checked that

- it commutes with $e_{i}, i \in\left\{i_{1}, \ldots, i_{t}\right\}$ if and only if $q$ is odd, and anticommutes otherwise,

- it commutes with $e_{i}, i \notin\left\{i_{1}, \ldots, i_{t}\right\}$ if and only if $q$ is even, and anticommutes otherwise.

Good candidates are of course the elements defined by

$$
p^{ \pm}=\prod_{e_{i}^{2}= \pm 1} e_{i}
$$

$p^{ \pm}=\prod e_{i}$ with $\prod$ extended over all $e_{i}^{2}= \pm 1$. Both of them satisfy $p^{2} \in \mathbb{R}$ and $p \bar{p} \in \mathbb{R}$, and so $m^{t}(p)= \pm m(\bar{p})= \pm m(p)$. Hence following cases are easily solved:

- If $s$ is even, put $\kappa=m\left(p^{+}\right)$.

- If $r$ is odd, put $\kappa=m\left(p^{-}\right)$.

It is also possible to determine wether the inner products defined are symmetric or antisymmetric. Indeed we have that

$$
\begin{aligned}
m^{t}\left(p^{+}\right) & =m^{t}\left(e_{1} \ldots e_{s}\right)=m^{t}\left((-1)^{s(s-1) / 2} e_{s} \ldots e_{1}\right) \\
& =(-1)^{s(s-1) / 2} m^{t}\left(e_{1}\right) \ldots m^{t}\left(e_{s}\right)=(-1)^{s(s-1) / 2} m\left(e_{1}\right) \ldots m\left(e_{s}\right),
\end{aligned}
$$

so the inner product will be symmetric for $s=0 \bmod 4$, while it will be antisymmetric for $s=2 \bmod 4, s$ being even. In a similar way

$$
\begin{aligned}
m^{t}\left(p^{-}\right) & =m^{t}\left(e_{s+1} \ldots e_{n}\right)=m^{t}\left((-1)^{r(r-1) / 2} e_{n} \ldots e_{s+1}\right) \\
& =(-1)^{r(r-1) / 2+r} m^{t}\left(e_{s+1}\right) \ldots m^{t}\left(e_{n}\right)=(-1)^{r(r+1) / 2} m\left(e_{s+1}\right) \ldots m\left(e_{n}\right),
\end{aligned}
$$

and the inner product defined is symmetric for $r=3 \bmod 4$ and antisymmetric for $r=1$ $\bmod 4$. 
It is possible that $m\left(p^{+}\right)= \pm m\left(p^{-}\right)$, so in the case $s$ even and $r$ odd we have not necessarily found two different metrics. This can only happen if both matrices are symmetric, or both antisymmetric, and this is the case if and only if $s+r=3 \bmod 4$ (and of course $s$ even and $r$ odd). Moreover $m\left(p^{+}\right)= \pm m\left(p^{-}\right)$implies that $m\left(p^{+} p^{-}\right)= \pm I$ which means that $m\left(\mathbb{R}_{s r}\right)$ is a non-universal Clifford algebra over $\mathbb{R}^{s r}$. But this is only possible if $r-s=3 \bmod 4\left(s\right.$ not necessarily even). So $m\left(p^{+}\right)= \pm m\left(p^{-}\right)$implies $s=0 \bmod 4$ and $r=3 \bmod 4$.

Matrices of a form similar to $m\left(p^{ \pm}\right)$were used in [4] and [5] to study Hurwitz pairs. It should be remarked however that the link between the inner product and the elements $p^{ \pm}$ only exists in the case of a standard representation, and condition (2) must be fulfilled.

This gives us one (or possibly two) Hurwitz pairs in the case $s$ even or $r$ odd. However from the table we can see that also for $s$ odd, $r$ even and $s-r=3 \bmod 4$ Hurwitz pairs exist. Moreover we have not found all possibilities, and we know nothing yet about equivalence of Hurwitz pairs.

The case $s-r=3 \bmod 4$ is easily solved. Here $\mathbb{R}_{s r}$ is isomorphic to $\mathbb{C}(\ell)$ for some $\ell$, either $\mathbb{R}_{s+1, r}$ or $\mathbb{R}_{s, r+1}$ is isomorphic to $\mathbb{R}(2 \ell)$. A metric derived from this extension can be used. Thet is, we extend the Clifford algebra with an element $e_{n+1}$, where $e_{n+1}^{2}=$ \pm 1 , depending on whether $\mathbb{R}_{s+1, r}$ or $\mathbb{R}_{s, r+1}$ is isomorphic to $\mathbb{R}(2 \ell)$ (and hence gives a representation over the same space as $\mathbb{R}_{s r}$. This bigger Clifford algebra has a Hurwitz pair, and the same inner product gives a Hurwitz pair for $\mathbb{R}_{s r}$.

Remark. The fact that for certain spaces $S$ no Hurwitz pairs exist is remarkable in view of the fact that there always exist (possibly reducible) representations such that inner product is kept. Indeed, if the the Clifford algebra $\mathbb{R}_{s r}$ over $S^{\prime}$ does not allow a Hurwitz pair, then $\mathbb{R}_{s+1, r}$ does allow a Hurwitz pair, and the restriction to $\mathbb{R}_{s r}$ of the representation satisfies H.1 and H.2. However the representation is not irreducible: it falls apart in two irreducible representations. Each of these must be isotropic spaces, since $\mathbb{R}_{s r}$ does not allow a Hurwitz pair. If, instead of dropping the newly added vector $e_{p+1}$, one drops $e$, we obtain a pair where conditions H.1 and H.3 are met. If one then applies the construction of section 1 to generate a new unit, one does not obtain a Hurwitz pair. Since H.1 and H.2 are satisfied, this means that H.3 must be violated, that is $V$ is split into two irreducible modules.

We now deal with the question of the type of the inner products for the Hurwitz pairs the existence of which was shown in the previous section. There are three possible types:

- the elliptic type, which is symmetric, $\sigma=1$, and which is of definite sign, that is $(v, v)_{K} \geq 0$ for all $v \in V$, or $(v, v)_{K} \leq 0$ for all $v \in V$,

- the hyperbolic type, which is also symmetric, but not of definite sign, and

- the symplectic type, which is antisymmetric.

There is an easy criterion to see whether a symmetric inner product will be elliptic or hyperbolic:

TheOREM 8. A symmetric inner product for a Hurwitz pair is elliptic if and only if $s=0$. 
Proof. Let $s \neq 0$. Then take $\vec{x}$ with $(\vec{x}, \vec{x})_{S}<0$ and $v$ with $(v, v)_{K} \neq 0$. Then $(\vec{x} v, \vec{x} v)_{K}=(\vec{x}, \vec{x})_{S}(v, v)_{K}$ has the opposite sign of $(v, v)_{K}$ itself and $K$ cannot be elliptic. Conversely, let $s=0$, take a standard representation $m$ and the $\kappa$ matrix for the inner product $K$. According to equation (1), $\kappa$ commutes with $m$, and so all eigenspaces of $\kappa$ are invariant under $m$. Since $m$ is irreducible, $V$ is an eigenspace of $\kappa$, and $K$ is elliptic.

In the sequel the difference between elliptic and hyperbolic metrics can thus be ignored, and the terms symmetric and antisymmetric will b used. Moreover we shall always assume a standard representation $m$. A metric whose matrix coincides (up to a scalar factor) with $m\left(p^{+}\right)$or $m\left(p^{-}\right)$will be called internal. The final theorem gives a complete classification of the possible Hurwitz pairs in the cases where at least one exists.

Theorem 9 (Classification theorem). Let $\left(\mathbb{R}_{s r}, V, m\right)$ be an irreducible representation, such that $m$ and $t$ are equivalent. then we have the following possibilities:

- $m\left(\mathbb{R}_{s r}\right)$ is a complete matrix algebra, $\mathbb{R}(\ell)$. Then the Hurwitz pair is up to equivalence unique.

- $m\left(\mathbb{R}_{s r}\right)$ is isomorphic to $\mathbb{C}(\ell)$ for some $\ell$, and there exists an internal Hurwitz pair. Then there are two Hurwitz pairs of different type.

- $m\left(\mathbb{R}_{\text {sr }}\right)$ is isomorphic to $\mathbb{C}(\ell)$ for some $\ell$, and there is no internal Hurwitz pair. Then there is (up to equivalence) a unique Hurwitz pair.

- $m\left(\mathbb{R}_{s r}\right)$ is isomorphic to $\mathbb{H}(\ell)$ for some $\ell$. Then there are two Hurwitz pairs, and they are of different type.

P r o of. We have already proved that at least one Hurwitz pair exists. According to to Lemma 7 we must find all matrices which commute with $m\left(\mathbb{R}_{s r}\right)$, and we must check whether there are equivalent pairs for the different $\kappa$ matrices we obtain.

- If $m\left(\mathbb{R}_{s r}\right)$ is isomorphic to $\mathbb{R}(\ell)$, then the only possibility for $\kappa_{j}^{-1} \kappa_{i}$ is, up to a constant the identity. Hence the Hurwitz pair is unique.

- If $m\left(\mathbb{R}_{s r}\right)$ is isomorphic to $\mathbb{C}(\ell)$, the matrices in $\mathbb{R}(2 \ell)$ commuting with $m\left(\mathbb{R}_{s r}\right)$ form a two dimensional vector space. This can be made explicit as follows: take the morphism $\mathbb{C}(\ell) \rightarrow \mathbb{R}(2 \ell)$ given by

$$
C \rightarrow\left(\begin{array}{cc}
\Re C & \Im C \\
-\Im C & \Re C
\end{array}\right),
$$

then it easily seen that the space of matrices commuting with the image of $\mathbb{C}(\ell)$ is spanned by the two matrices

$$
I=\left(\begin{array}{cc}
I_{\ell} & 0 \\
0 & I_{\ell}
\end{array}\right) \quad \text { and } \quad J=\left(\begin{array}{cc}
0 & I_{\ell} \\
-I_{\ell} & 0
\end{array}\right)
$$

(which are of course the algebra images of 1 and $i$ ). It has been proved in chapter I that $J= \pm m\left(e_{N}\right)$, and so for a standard representation $J^{t}=-J$. Thus, starting from a given $\kappa$ we obtain a two dimensional vector space of possible matrices $M$ satisfying $\operatorname{Mm}(\vec{x})=$ $-m^{t}(\vec{x}) M$. Assume now that $\kappa_{1}$ is internal. It then follows that $J$ commutes with $\kappa_{1}$, and we put $\kappa_{2}=J \kappa_{1}$. If $\kappa_{1}^{t}=\sigma_{1} \kappa_{1}$, one obtains that $\kappa_{2}^{t}=\kappa_{1}^{t} J^{t}=-\sigma_{1} \kappa_{1} J=-\sigma_{1} \kappa_{2}$, since $J$ is antisymmetric and commutes with $\kappa_{1}$. Thus $\kappa_{1}$ and $\kappa_{2}$ are of different type and cannot lead to equivalent Hurwitz pairs. 
If on the other hand no internal Hurwitz pair exists, we still have obtained a $\kappa_{1}$. This anticommutes with $J$. Now all matrices $C$ which commute with the representations of the Clifford algebra are of the form $C=\lambda I+\mu J$ for real $\lambda$ and $\mu$, and since $J^{2}=-I$ this gives a representation of $\mathbb{C}$ where complex conjugation coincides with taking the transpose. It follows that $C$ is invertible if and only if $\lambda^{2}+\mu^{2} \neq 0$ and that

$$
\begin{aligned}
C^{-t} \kappa_{1} & C^{-1} \\
& =\left(\lambda^{2}+\mu^{2}\right)^{-2}(\lambda I+\mu J) \kappa_{1}(\lambda I-\mu J) \\
& =\left(\lambda^{2}+\mu^{2}\right)^{-2}(\lambda I+\mu J)^{2} \kappa_{1} .
\end{aligned}
$$

The set of $\kappa$ matrices for which the Hurwitz pair is equivalent to the first one is hence two dimensional, and all Hurwitz pairs are equivalent.

- If $F=\mathbb{H}$ a similar proof applies. The set of matrices commuting with the representation is four dimensional, and gives a representation of $\mathbb{H}$, where again conjugation coincides with transposition. There is a basis of the form $\{I, J, K, J K\}$, with the obvious link to the quaternions. Let $\kappa_{1}$ stand for the internal Hurwitz pair. In a way similar to the one for the complex case it is proved that any matrix $(\mu J+\nu K+\rho J K) \kappa_{1}$ is of different type than $\kappa_{1}$ itself. We now prove that all such matrices lead to equivalent Hurwitz pairs. We prove that all are equivalent to $J \kappa_{1}$. We obtain for $C=\lambda I+\mu J+\nu K+\rho J K$

$$
\begin{aligned}
\left(\lambda^{2}+\mu^{2}+\nu^{2}+\rho^{2}\right)^{2} & C^{-t} J \kappa_{1} C^{-1} \\
& =(\lambda I+\mu J+\nu K+\rho J K) J \kappa_{1}(\lambda I-\mu J-\nu K-\rho J K) \\
& =(\lambda I+\mu J+\nu K+\rho J K)(\lambda I-\mu J+\nu K+\rho J K) J \kappa_{1} \\
& =\left[\left(\lambda^{2}+\mu^{2}-\nu^{2}-\rho^{2}\right)^{-2} J\right. \\
& =-(2 \lambda \nu-2 \mu \rho) J K+(2 \lambda \rho+2 \mu \nu) K] \kappa_{1},
\end{aligned}
$$

so the set of matrices equivalent to $J \kappa_{1}$ is three dimensional.

COROLlary 10. Two Hurwitz pairs over the same representation of a Clifford algebra which are of the same type are equivalent.

Summarising, the construction goes as follows. First the internal Hurwitz pairs are considered:

- If $s=0 \bmod 4$, there is a symmetric Hurwitz pair.

- If $s=2 \bmod 4$, there is an antisymmetric Hurwitz pair.

- If $r=1 \bmod 4$, there is an antisymmetric Hurwitz pair.

- If $r=3 \bmod 4$, there is a symmetric Hurwitz pair.

This completes the internal Hurwitz pairs. The external ones are given by:

- If the representation is isomorphic to $\mathbb{C}(\ell)$ or $\mathbb{H}(\ell)$ and there is an internal Hurwitz pair of one type, there is also a representation (which can be external or internal) of the other type.

- If $r-s=1 \bmod 4, s$ is odd and $r$ even, then one looks up in the Clifford table whether $\mathbb{R}_{s, r+1}$ or $\mathbb{R}_{s+1, r}$ is isomorphic to $\mathbb{R}(2 \ell)$. The Hurwitz pair is inherited from this algebra. 


\section{Clifford valued inner products}

Inner product modules. An inner product module $H$ over $\mathbb{R}_{p q}$ is a (left) module over $\mathbb{R}_{p q}$ with a real valued inner product $(\cdot, \cdot)_{\mathbb{R}}$ satisfying (for all $x$ and $y$ in $H$ and $\lambda$ in $\mathbb{R}_{p q}$ )

$$
(x, \lambda y)_{\mathbb{R}}=(\bar{\lambda} x, y)_{\mathbb{R}} .
$$

This is the compatibility condition stating that the operation of taking the adjoint gives the main antiautomorphism when restricted to the Clifford algebra. The product is called non-degenerate if the inner product is non-degenerate. Inner products will be assumed to be non-degenerate unless explicitly stated otherwise. The inner product can be symmetric or antisymmetric, or none of the two.

A Clifford inner product module $H$ over $\mathbb{R}_{p q}$ is a (left) module over $\mathbb{R}_{p q}$ with an $\mathbb{R}_{p q}$ valued inner product $(\cdot, \cdot)$ satisfying (for all $x, y$ and $z$ in $H$ and $\lambda$ in $\mathbb{R}_{p q}$ )

(i) $(x+\lambda y, z)=(x, z)+\lambda(y, z)$

(ii) $H$ with the real valued inner product $(\cdot, \cdot)_{\mathbb{R}}$ defined by $(x, y)_{\mathbb{R}}=\Re(x, y)$ is an inner product module.

The inner product is called symmetric if $(x, y)=\overline{(y, x)}$, antisymmetric if $(x, y)=$ $-\overline{(y, x)}$.

TheOREM 11. Each inner product module over $\mathbb{R}_{p q}$ can be considered as a Clifford inner product module. The Clifford inner product is symmetric (antisymmetric) if and only if the real inner product is.

Before we prove this the following remark should be made: if $T \in L\left(\mathbb{R}_{p q}, \mathbb{R}\right)$ then there exists a unique element $\phi(T)$ of the Clifford algebra such that

$$
T(\lambda)=\Re(\lambda \phi(T))
$$

for all $\lambda \in \mathbb{R}_{p q}$. The mapping $\phi$ is one-to-one.

Proof. We have to prove that the inner product $(\cdot, \cdot)_{\mathbb{R}}$ can be extended to a Clifford valued inner product $(\cdot, \cdot)$. This can be done as follows: take $x$ and $y$ in $H$ arbitrary. Then the mapping $T: \mathbb{R}_{p q} \rightarrow \mathbb{R}$ defined by

$$
T_{x, y}(\lambda)=(\lambda x, y)
$$

is a real linear map, i.e. $T_{x, y} \in L\left(\mathbb{R}_{p q}, \mathbb{R}\right)$. We define $(x, y)$ by

$$
(x, y)=\phi\left(T_{x, y}\right) \text {. }
$$

We get

(ia) $(x, y)+(x, z)=(x, y+z)$ (trivial).

(ib) $(\lambda x, y)=\lambda(x, y)$. Indeed, from the definition we have that for $\mu$ arbitrary

$$
(\mu \lambda x, y)_{\mathbb{R}}=\mu \phi\left(T_{\lambda x, y}\right)=\mu \lambda \phi\left(T_{x, y}\right)
$$

so $\phi\left(T_{\lambda x, y}\right)=\lambda \phi\left(T_{x, y}\right)$, or $(\lambda x, y)=\lambda(x, y)$.

(ii) That $(x, y)_{\mathbb{R}}=\Re(x, y)$ is obvious.

We now prove that if the real inner product is symmetric, so is the Clifford valued inner product (the proof for the antisymmetric case is similar). Assume $(,)_{\mathbb{R}}$ is symmetric. then we have for $\lambda, x$ and $y$ arbitrary that

$$
(\lambda x, y)_{\mathbb{R}}=\Re(\lambda(x, y))
$$


while on the other hand

$$
(\lambda x, y)_{\mathbb{R}}=(x, \bar{\lambda} y)_{\mathbb{R}}=(\bar{\lambda} y, x)_{\mathbb{R}}=\Re(\bar{\lambda}(y, x)) .
$$

Since the equation $\Re(\lambda(x, y))=\Re(\bar{\lambda}(y, x))$ holds for any $\lambda$ it follows that $(y, x)=\overline{(x y)}$.

It is possible to give an explicit formulation of the Clifford valued inner product in terms of the real valued inner product as follows:

$$
(x, y)=\sum_{A} e_{A}^{-1}\left(e_{A} x, y\right)_{\mathbb{R}}
$$

Hurwitz pairs. Since a Hurwitz pair can be considered as an irreducible representation of a Clifford algebra, hence as a minimal left ideal of the algebra, it is closely linked with primitive idempotents, since each minimal ideal is generated by a primitive idempotent.

Let $J$ be such a primitive idempotent of $\mathbb{R}_{p q}$ and $I_{J}$ the minimal ideal

$$
I_{J}=\left\{a J: a \in \mathbb{R}_{p q}\right\} .
$$

If $\mathbb{R}_{p q}$ allows a Hurwitz pair then $I_{J}$ can be given a non-degenerate inner product such that the compatibility condition is satisfied, and so can be turned into a Clifford inner product module. This inner product is completely defined by the value of $(J, J)$, since $(a J, b J)=a(J, J) \bar{b}$ for any $a, b \in \mathbb{R}_{p q}$. On the other hand, if $\lambda$ is an arbitrary element of $\mathbb{R}_{p q}$, then one can define an inner product on $I_{J}$ putting $(a J, b J)_{\lambda}=a J \lambda \bar{J} \bar{b}$, which can be degenerate, and even be zero.

THEOREM 12. Following statements are equivalent:

- There exists a Hurwitz pair for $\mathbb{R}_{p q}$

- given an arbitrary primitive idempotent $J$ there exists a $\lambda$ in $\mathbb{R}_{p q}$ such that $J \lambda \bar{J}$ is different from zero.

Proof. If there is a Hurwitz pair, the existence of a $\lambda$ satisfying the condition is obvious.

Suppose now such $\lambda$ exists. We have to prove that the inner product $\Re(\cdot, \cdot)_{\lambda}$ is not degenerate on $I_{J}$, in other words that for any $a$ such that $a J \neq 0$ there exists a $b$ such that $\Re(b J, a J)_{\lambda} \neq 0$. We first prove that $a J \bar{\lambda} \bar{J} \neq 0$ for any $a$ with $a J \neq 0$. The set

$$
Z=\{a: a J \bar{\lambda} \bar{J}=0\}
$$

is a left ideal of $\mathbb{R}_{p q}$, which is proper, since evidently $1 \notin Z$. Moreover it contains the ideal $Z_{J}=\{a: a J=0\}$. But the latter is a maximal ideal, so $Z=Z_{J}$ and $a J \lambda \bar{J}=0$ implies $a J=0$. Take now arbitrary $a$ such that $a J \neq 0$. Then $a J \bar{\lambda} \bar{J} \neq 0$, and also $J \lambda \bar{J} \bar{a} \neq 0$. Since the mapping $\phi^{-1}: \mathbb{R}_{p q} \rightarrow L\left(\mathbb{R}_{p q}, \mathbb{R}\right)$ is one-to-one, $\phi^{-1}(J \lambda \bar{J} \bar{a})$ is not the zero functional, and so there exists $b$ such that $\phi^{-1}(J \lambda \bar{J} \bar{a})(b)=\Re(b J \lambda \bar{J} \bar{a}) \neq 0$, in other words $\Re(b J, a J)_{\lambda} \neq 0$. This proves that the inner product $(\cdot, \cdot)_{\lambda}$ is non-degenerate, and that a Hurwitz pair exists.

It is easy to see whether the inner product defined by $\lambda$ is symmetric or antisymmetric since

$$
\begin{aligned}
& (a J, b J)_{\lambda}=a J \lambda \bar{J} \bar{b} \\
& \overline{(b J, a J)_{\lambda}}=a J \bar{\lambda} \bar{J} \bar{b} .
\end{aligned}
$$


So the inner product is symmetric or antisymmetric if and only if $J \lambda \bar{J}= \pm J \bar{\lambda} \bar{J}$. Of course in these cases we can take $\lambda$ such that $\lambda= \pm \bar{\lambda}$.

This construction gives an alternative way to classify Hurwitz pairs, and one in which matrix representations are no longer needed. Indeed it is sufficient to take for a given Clifford algebra an arbitrary primitive idempotent, and to see whether $J \mathbb{R}_{p q} \bar{J}=\{0\}$. by

As an example we take the Clifford algebra $\mathbb{R}_{n, n}$. A primitive idempotent here is given

$$
J=\prod_{j=1}^{n} \frac{1}{2}\left(1+e_{j} e_{n+j}\right) .
$$

If $\lambda$ commutes with any factor $\left(1+e_{j} e_{n+j}\right)$ then $\left(1+e_{j} e_{n+j}\right) \lambda\left(1-e_{j} e_{n+j}\right)=0$, which implies that $J \lambda \bar{J}=0$. So

$$
J \mathbb{R}_{p q} \bar{J}=\left\{J\left(\prod_{j=1}^{n}\left(a_{j} e_{j}+b_{j} e_{n+j}\right)\right) \bar{J}\right\} .
$$

For $\lambda=\prod_{j=1}^{n}\left(a_{j} e_{j}+b_{j} e_{n+j}\right)$ we have that $\lambda=\bar{\lambda}$ if $n \bmod 4=0$ or 3 , so we have a symmetric inner product there, and $\lambda=-\bar{\lambda}$ if $n \bmod 4=1$ or 2 , which gives an antisymmetric inner product.

To provide the link between the $\lambda$ defined inner product and the one determined by $\kappa$ it is useful to introduce a fairly uncommon expression for the second inner product. Let $x$ and $y$ be coordinate column vectors associated with the representation $m$, and let $u$ be the coordinate vector of the primitive idempotent $J$. The inner product defined by $\kappa$ can be expressed as

$$
(x, y)_{\kappa}=x^{t} \kappa y=\sum_{i, j} x_{i} \kappa_{i j} y_{j}=\sum_{i, j}\left(x_{i} y_{j}\right) \kappa_{j i}^{t}=\operatorname{tr}\left(\left(x y^{t}\right) \kappa^{t}\right) .
$$

Moreover the inner product is completely determined from the expression $\left(x y^{t}\right) \kappa^{t}$ for any fixed non zero $x$ and $y$, since for any $a$ and $b$ in the Clifford algebra the equality $(m(a) x, m(b) y)_{\kappa}=(m(\bar{b}) m(a) x, y)_{\kappa}=\operatorname{tr}\left(m(\bar{b}) m(a)\left(x y^{t}\right) \kappa^{t}\right)$ holds. The most straightforward choice is $x=y=u$, leading to

$$
(m(a) u, m(b) u)_{\kappa}=\operatorname{tr}\left(m(\bar{b}) m(a)\left(u u^{t}\right) \kappa^{t}\right)=\operatorname{tr}\left(m(\bar{b} a J)\left(u u^{t}\right) \kappa^{t}\right)
$$

In order to find a $\lambda$ such that $\Re(a J, b J)_{\lambda}=(m(a) u, m(b) u)_{\kappa}$ the equation

$$
\operatorname{tr}\left(m(\bar{b} a J)\left(u u^{t}\right) \kappa^{t}\right)=\frac{1}{t} \operatorname{tr}(m(\bar{b} a J) m(\lambda)
$$

should hold where $t=\operatorname{dim} V$. (Notice that the equality $\Re a=\frac{1}{t} \operatorname{tr}(m(a))$ of course does not hold for all $a$ of the Clifford algebra. As an example we have in the case $p-q=1 \bmod 4$ the pseudo scalar, for which $m\left(e_{N}\right)= \pm I$. It does hold however for $a$ in the left ideal $\left.\mathbb{R}_{s r} \bar{J}\right)$. Let now $\langle A, B\rangle=\frac{1}{t} \operatorname{tr} A B^{t}$ be the inner product of the matrix algebra considered (this is a real matrix algebra, so no conjugation is needed). If we can prove that $m^{t}(\lambda)$ is of the form $A m(J)$ for some matrix $A$, then we have that $m^{t}(\lambda)$ must be the orthogonal projection of $\kappa\left(u u^{t}\right)$ on the ideal $m\left(\mathbb{R}_{s r} J\right)$ of the matrix algebra. But $m^{t}(\lambda)=m^{t}(J \lambda)=$ $m^{t}(\lambda) m^{t}(J)$, so it is sufficient to prove that $m(J)=m^{t}(J)$. To do this we start from the equality $m^{t}\left(e_{i}\right)=e_{i}^{2} m\left(e_{i}\right)$. it follows that $m^{t}\left(e_{i}\right) m^{t}\left(e_{j}\right)=e_{i}^{2} e_{j}^{2} m\left(e_{i}\right) m\left(e_{j}\right)$ and hence that 
$m^{t}\left(e_{j}\right) m^{t}\left(e_{i}\right)=m^{t}\left(e_{i j}\right)=\left(e_{i j}\right)^{2} m\left(e_{i j}\right)$. By induction then $m^{t}\left(e_{A}\right)=e_{A}^{2} m\left(e_{A}\right)$. Since $J$ is composed of commuting factors the form $\frac{1}{2}\left(1+e_{A}\right)$ where $e_{A}^{2}=1$, the proposed equality follows.

\section{References}

[1] F. Brackx, R. Delanghe and F. Sommen, Clifford analysis, Pitman, London, 1982.

[2] A. Hurwitz, Über die Komposition der quadratischen Formen von beliebig vielen Variablen, Nachrichten von der Königlichen Gesellschaft der Wissenschaften zu Göttingen Math. phys. Kl. (1898), 308-316, reprinted in: A. Hurwitz, Mathematische Werke II, Birkhäuser Verlag, Basel, 1933, 565-571.

[3] A. Hurwitz, Über die Komposition der quadratischen Formen, Math. Ann. 88 (1923), 1-25, reprinted in: A. Hurwitz, Mathematische Werke II, Birkhäuser Verlag, Basel, 1933, 641-666.

[4] J. Ławrynowicz and J. Rembieliński, Pseudo-euclidean Hurwitz pairs and generalized Fueter equations, in J. S. R. Chisholm and A. K. Common (eds): Clifford algebras and their applications in mathematical physics, D. Reidel Publ. Co. Dordrecht, 1986, 39-48.

[5] J. Ławrynowicz and J. Rembieliński, Complete classification for pseudo-euclidean Hurwitz pairs including symmetry applications, Bull Soc. Sci. Lettres Łódź 36, No. 29, 1986, 15 pp.

[6] P. Lounesto, Clifford algebras and spinors, in J. S. R. Chisholm and A. K. Common (eds): Clifford algebras and their applications in mathematical physics, D. Reidel Publ. Co. Dordrecht, 1986, 25-37.

[7] I.R. Porteous, Topological geometry, 2nd edition, Cambridge University Press, 1981.

[8] I.R. Porteous, Clifford algebra tables, in F. Brackx, R. Delanghe and H. Serras (eds.) Clifford algebras and their applications in mathematical physics, Kluwer, Dordrecht, 1993, $13-22$.

[9] E. Ramirez de Arellano, M. Shapiro and N. Vasilevski, Hurwitz pairs and Clifford algebra representations, in F. Brackx, R. Delanghe and H. Serras (eds.) Clifford algebras and their applications in mathematical physics, Kluwer, Dordrecht, 1993, 175-182.

[10] L.-S. Randriamihamison, Paires de Hurwitz pseudo-euclidiennes en signature quelconque, J. Phys. A: Math. Gen. 23 (1990), 2729-2749. 